\title{
Preparation of PVDF/PMMA Blend Nanofibers by Electrospray Deposition: Effects of Blending Ratio and Humidity
}

\author{
By Muhamad NASIR, ${ }^{1}$ Hidetoshi MATSUMoto,${ }^{1}$ Mie MinaGaWA, ${ }^{1}$ \\ Akihiko TANIOKA, ${ }^{1, *}$ Tetsuya DANNO, ${ }^{2}$ and Hideo HORIBE ${ }^{3}$
}

Poly(vinylidene fluoride) (PVDF)/poly(methylmethacrylate) (PMMA) blend nanofibers were prepared by electrospray deposition (ESD). To control the diameter, morphology, and crystalline structure of nanofiber, the effects of the blending ratio and the humidity during ESD were investigated. The increase in the PVDF blending ratio reduced the average fiber diameter and enhanced formation of crystalline structure. In particular, ESD process enhanced crystal formation of PVDF in high-PVDF blending ratio. High humidity reduced the formation of beaded fiber and increased the average fiber diameter. These results provide fundamental information for preparation of the smooth and crystalline PVDF/PMMA blend nanofiber by ESD.

KEY WORDS: Electrospray Deposition / PVDF/PMMA Blend Nanofiber / Humidity / Crystalline Structure /

Recently, nanofibers have been gained much attention in the research fields of science and technology. ${ }^{1-3}$ The properties and performances of nanofiber can be improved by controlling the morphology, fiber diameter, and crystalline structure of nanofiber. For example, by reducing fiber diameter down to nanometer-scale, we can produce the nanofibers with a large specific surface area, which improve the adsorption and filtration properties (e.g., slip flow effect in air filtration). ${ }^{4-7}$

Electrospray deposition (ESD) process is a versatile method for producing nanofiber. ${ }^{8}$ Poly(vinylidene fluoride) (PVDF) is a semi-crystalline polymer which has excellent chemical, electrical, and mechanical properties. PVDF have two main crystalline structures such as $\alpha$ - and $\beta$-phases. The $\alpha$-phase crystal has nonpolar properties while the $\beta$-phase one has polar properties. ${ }^{9,10}$ Theoretical study revealed that $\alpha$-phase crystal is more thermodynamically stable than $\beta$-phase one. Our previous study, however, showed that the ESD process enhanced the formation of $\beta$-phase crystalline structure of PVDF nanofiber. ${ }^{8}$ The electret fibers due to their polar structure can enhance the collection efficiency for both the charged and uncharged aerosols in filtration. ${ }^{11}$ In addition, the $\beta$-phase crystalline PVDF with piezoelectricity is very important material for sensors and actuators. Poly(methylmethacrylate) (PMMA) is an amorphous polymer and has a good miscibility with PVDF in melt. The physical properties of the PVDF/PMMA blend have been intensively studied. ${ }^{12-14}$ The blending composition is a crucial factor for controlling the crystallinity and the crystalline structure of PVDF in the blend. Blending of PVDF with PMMA also combine the advantages of PVDF and PMMA and enhance the economic value of the PVDF-based materials.
The objectives of the present study are to prepare PVDF/ PMMA blend nanofibers by ESD and to investigate the effects of blending ratio and humidity on morphology, average fiber diameter, and crystalline structure of the nanofiber.

\section{EXPERIMENTAL}

\section{Materials}

PVDF with $M_{\mathrm{w}}=141,000$ and $M_{\mathrm{n}}=64,000$ (Kureha, Japan), and PMMA with $M_{\mathrm{w}}=168,000$ and $M_{\mathrm{n}}=96,400$ (Mitsubishi Chemical, Japan) were used. PVDF/PMMA blends were prepared by milling the PVDF and PMMA pellet with a uniaxial melt extruder. ${ }^{12}$ The blending ratio of PVDF/ PMMA is $20 / 80,40 / 60,50 / 50,70 / 30,80 / 20$, and 90/10 w/w. $N, N$-dimethyl acetamide (DMAc) (Wako, Japan) was used as solvent. The dry nitrogen gas and inorganic salts such as $\mathrm{NaCl}$ and $\mathrm{K}_{2} \mathrm{SO}_{4}$ (Wako, Japan) were used to control humidity.

\section{Preparation of PVDF/PMMA Belend Solutions}

PVDF/PMMA blend at various blending ratios were dissolved in DMAc, stirred at $60^{\circ} \mathrm{C}$ for several hours, and then cooled to room temperature before use. The total polymer blend concentration was optimized to obtain smooth (beadfree) fiber. Smooth fiber was obtained at polymer blend concentration higher than $26 \mathrm{wt} \%$. Surface tension and viscosity of solution were measured by a digital surface tensiometer (CBVP-Z, Kyowa Interface Science, Japan) and a digital viscometer (VM-100A, CBC Materials, Japan), respectively.

\footnotetext{
${ }^{1}$ Department of Organic and Polymeric Materials, and International Research Center of Macromolecular Science, Tokyo Institute of Technology, Mail Box S8-27, 2-12-1 Ookayama, Meguro-ku, Tokyo 152-8552, Japan

${ }^{2}$ Department of Lifestyle Design, Kochi Prefectural University, 5-15 Eikokuji-cho, Kochi 783-8015, Japan

${ }^{3}$ Research Laboratory of Integrated Technology Systems, Kanazawa Institute of Technology, 3-1 Yatsukaho, Hakusan 924-0838, Japan

*To whom correspondence should be addressed (Tel: +81-3-5734-2426, Fax: +81-3-5734-2876, E-mail: tanioka.a.aa@m.titech.ac.jp).
} 


\section{Preparation of PVDF/PMMA Blend Nanofiber}

A polymer blend solution was placed in a $1-\mathrm{mL}$ syringe which was attached to a syringe-type infusion pump (MCIP III, Minato Concept, Japan). The stainless-steel needle (inner diameter $=1 \mathrm{~mm}$ ) of syringe was connected to a high voltage regulated DC power supply (HDV-20K75STD, Pulse Electronic Engineering, Japan). The detailed procedure for preparation of nanofiber by $\mathrm{ESD}$ can be found in our previous paper. ${ }^{8}$ Both the saturated inorganic salt solutions such as $\mathrm{Na}_{2} \mathrm{SO}_{4}$ and $\mathrm{NaCl}^{15}$ and the dry nitrogen gas were used to control the humidity during ESD. The nozzle was placed in the sealed chamber containing the saturated salt solution bath. In addition, the chamber was connected to nitrogen gas tank. After ESD, nanofibers were vacuum-dried for several hours to remove the residual of solvent.

\section{Characterization of PVDF/PMMA Nanofiber}

Morphology of nanofiber was observed by a scanning electron microscope (SEM, SM-200, Topcon, Japan). All samples were sputter-coated with Au. SEM image of nanofiber were used to analyze morphology of nanofiber and to calculate fiber diameters by using Photoshop 7.0 (Adobe, USA).

\section{Characterization of Crystallinity and Crystal Structure of PVDF/PMMA Nanofiber}

The degree of crystalinity and melting point were determined by differential scanning calorimetry with heating rate of $10^{\circ} \mathrm{C} / \mathrm{min}$ (DSC 6100 , Seiko Instruments, Japan). The crystalline structure of PVDF in the PVDF/PMMA nanofibers were characterized by a Fourier transform infrared spectrometer (FT-IR, FT/IR-410, Jasco, Japan) and a wide angle X-ray diffraction (WAXD) meter (MiniFlex, Rigaku, Japan). ${ }^{8,12-14}$

\section{RESULT AND DISCUSSION}

\section{Effect of PVDF/PMMA Blending Ratio on Nanofiber Formation}

Figure 1 shows the effect of blending ratio on viscosity and surface tension of PVDF/PMMA solution. Viscosity of the solution decreased with the increase in the PMMA ratio. The surface tension of the solution was almost constant in the range of the PVDF/PMMA ratio between 20/80 and 70/30, while at the higher PVDF ratio, the surface tension decreased with the increase in the PVDF ratio.

Figures 2 and 3 are surface micrographs and average fiber diameter of PVDF/PMMA nanofibers with various blending ratios, respectively. In general, a more viscous polymer solution tends to form thicker fiber. The average fiber diameter, however, increased with the increase in the PMMA ratio even if viscosity of solution was decreased. This trend can be explained by the difference of electrospinnability of each polymer component in the blend solution.

\section{Effect of Humidity}

Figures 3 and 4 show the effect of blending ratio and humidity on average diameter of the PVDF/PMMA blend

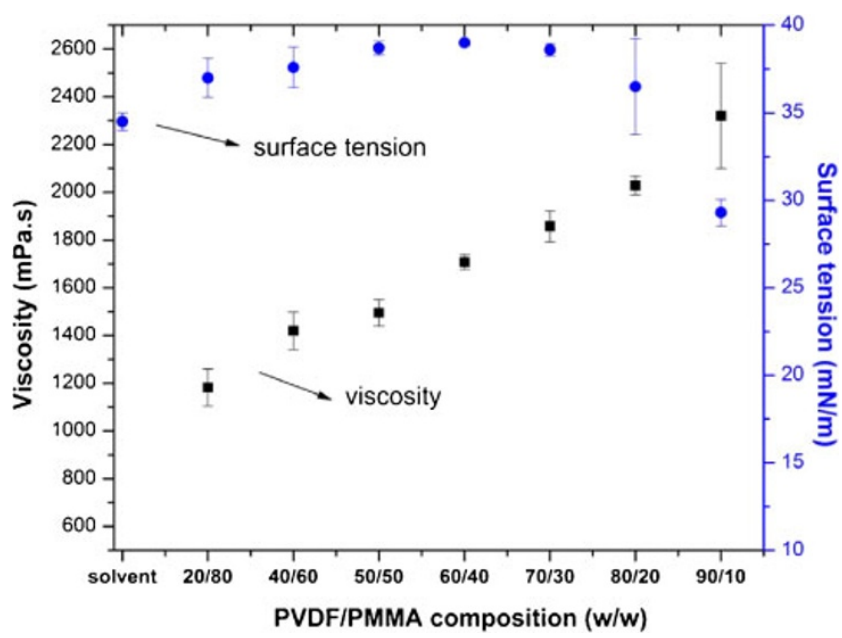

Figure 1. Effect of PVDF/PMMA blending ratio on viscosity and surface tension of polymer solutions (concentration of PVDF/PMMA solution $=26 \mathrm{wt} \%$ )
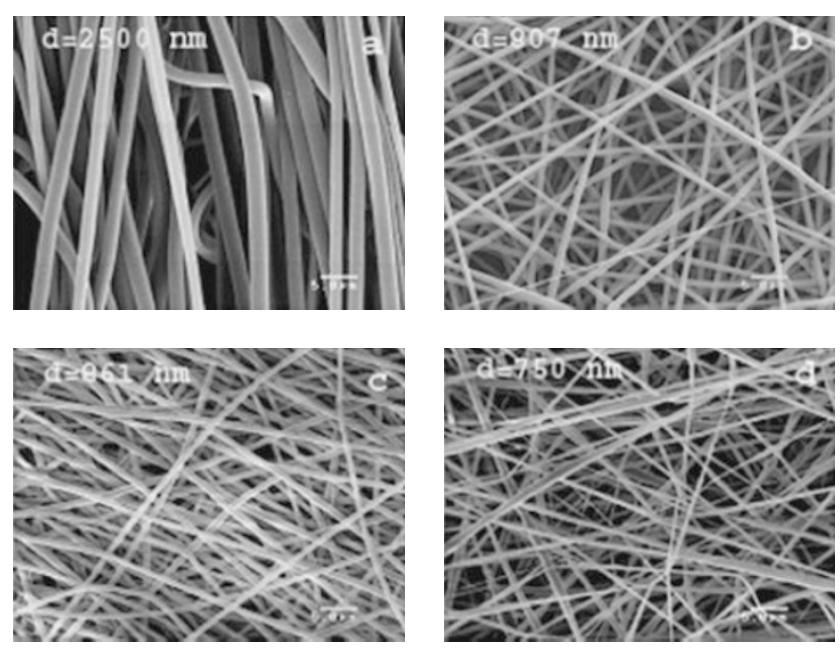

Figure 2. Morphology of PVDF/PMMA nanofibers with various blending ratio; PVDF/PMMA = (a) 20/80, (b) 50/50, (c) 60/40, and (d) $90 /$ $10 \mathrm{w} / \mathrm{w}$. ESD condition: polymer concentration $(\mathrm{C})=30 \mathrm{wt} \%$, The nozzle-to-collector distance $(\mathrm{d})=12 \mathrm{~cm}$, applied voltage $(\mathrm{V})=15$ $\mathrm{kV}$, flow rate $(\mathrm{Q})=2 \mu \mathrm{L} / \mathrm{min}$, and humidity $(\mathrm{H})=25-30 \%$.

nanofibers. Figure 3 shows that the average diameter of all nanofibers was influenced by humidity. In all blending ratios, the increase in humidity increased fiber diameter. In Figure 4, at very low humidity $(\mathrm{RH} \sim 15 \%)$, fibers with average diameter around $400 \mathrm{~nm}$ were formed, while at high humidity ( $\mathrm{RH}>50 \%$ ), the microscaled fiber was formed.

Figure 5 shows the morphology of PVDF/PMMA blend nanofibers prepared under various humidity conditions. The increase in humidity decreased the amount of "bead on the fiber," while the higher humidity than 50\%, "fused fibers" were formed. Vrieze et al., have showed that humidity makes the nanofibers thicker or thinner, depending on the chemical nature of polymer. ${ }^{15}$ In this case, absorption of surrounding water causes the polymer jet to solidify faster. The elongation of the jet due to the electrostatic repulsive force cannot 


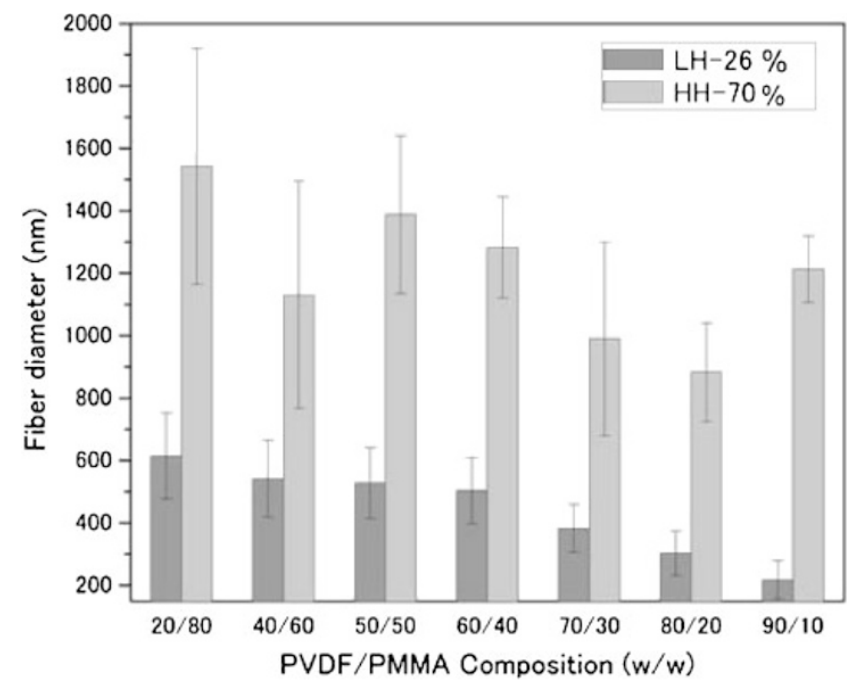

Figure 3. Effect of PVDF/PMMA blending ratio and humidity on fiber diameter. $\mathrm{LH}-26 \%=$ low humidity $(26 \%), \mathrm{HH}-70 \%=$ high humidity $(70 \%)$ and polymer blend concentration $=26 \mathrm{wt} \%$.

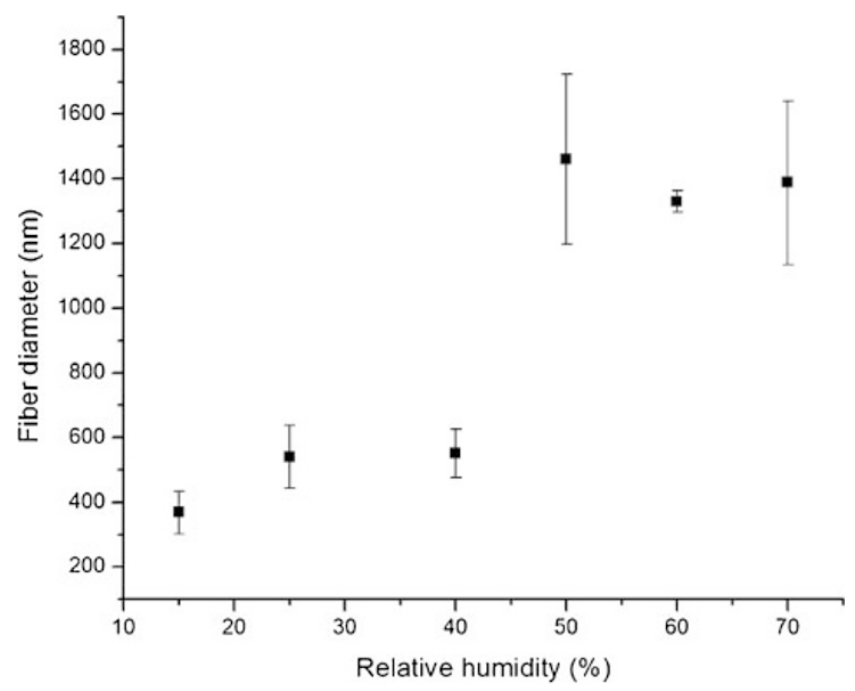

Figure 4. Effect of relative humidity on fiber diameter (PVDF/PMMA blending ratio $=50 / 50 \mathrm{w} / \mathrm{w}$ ).

continue longer, resulting in thicker fibers. In addition, since surrounding water prevents evaporation of the solvent from the polymer jet, the remaining solvent in the as-deposited fibers cause the fibers to fuse together.

\section{Crystallinity and Crystal Structure of PVDF/PMMA Blend Nanofiber}

Table I shows the degree of crystallinity and melting point of the PVDF/PMMA belnd nanofiber determined by the DSC measurements. At the low PVDF ratio (20 wt \%), the PVDF/ PMMA blend nanofiber exhibited dominantly amorphous state and the crystallinity was very low. These results showed that higher content of PMMA in the PVDF/PMMA blend inhibited the crystallization of PVDF. Similar results were reported by Horibe et al. for the PVDF/PMMA bulk blend by quenching
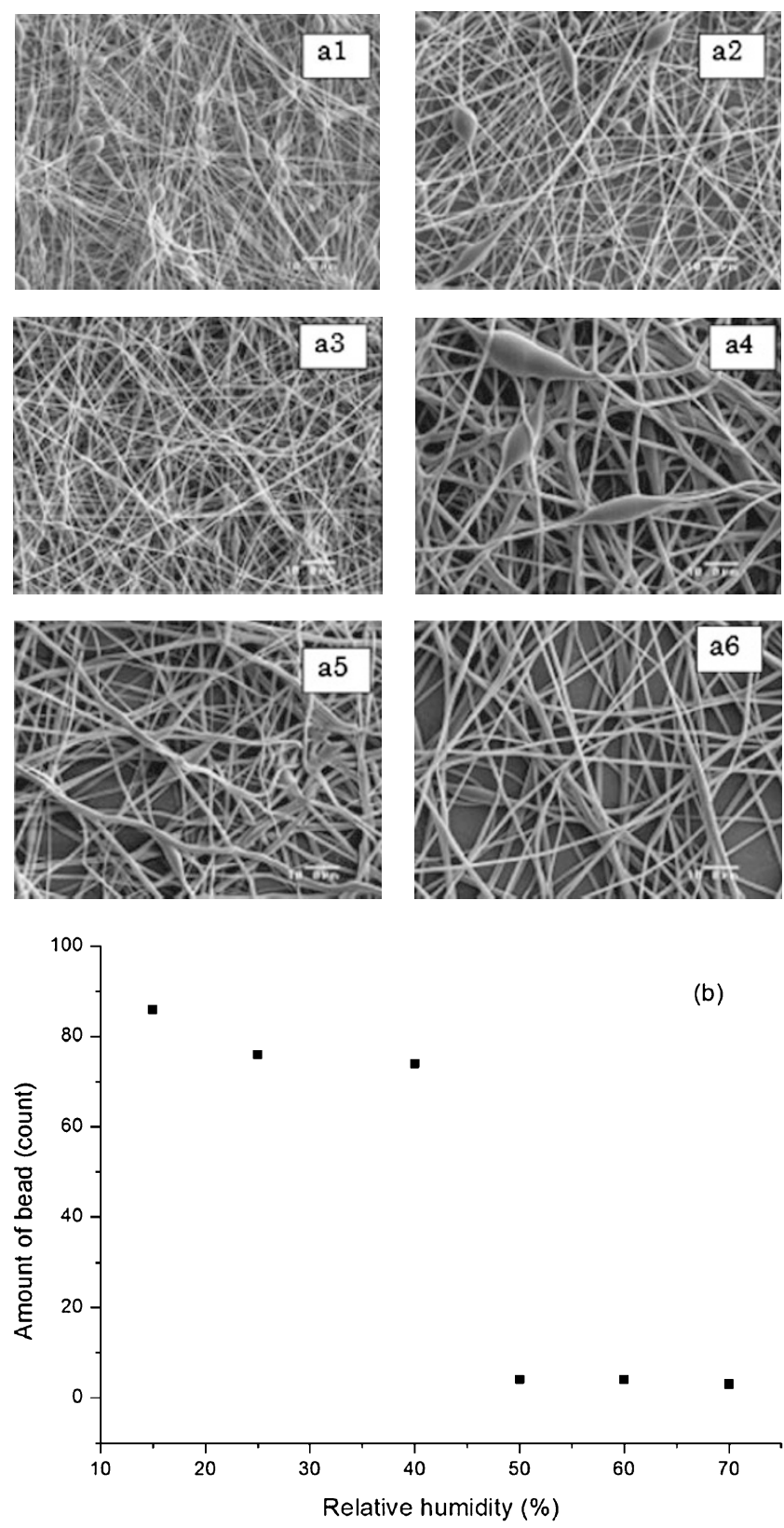

Figure 5. Effect of the humidity on the morphology and the amount of bead on the fiber. (a) Morphology of the PVDF/PMMA blend nanofiber prepared under various humidity conditions: $\mathrm{RH}=(\mathrm{a}) 15 \%$, (a2) $25 \%$, (a3) $40 \%$, (a4) 50\%, (a5) $60 \%$, and (a6) $70 \%$. (b) The amount of beads on the fiber at the various humidity conditions.

Table I. Total crystallinity of PVDF/PMMA blend nanofiber

\begin{tabular}{cccc}
\hline Sample & $\begin{array}{c}\text { Total degree } \\
\text { of crystallinity } \\
(\%)\end{array}$ & $\begin{array}{c}\text { Reduced degree } \\
\text { of crystallinity } \\
(\%)\end{array}$ & $\begin{array}{c}\text { Melting } \\
\text { temperature } \\
\left({ }^{\circ} \mathbf{C}\right)\end{array}$ \\
\hline PVDF/PMMA $=20 / 80$ & Amorphous & Amorphous & - \\
PVDF/PMMA $=50 / 50$ & 20 & 41 & 159 \\
PVDF/PMMA $=60 / 40$ & 35 & 59 & 168 \\
PVDF/PMMA $=90 / 10$ & 55 & 62 & 174 \\
\hline
\end{tabular}




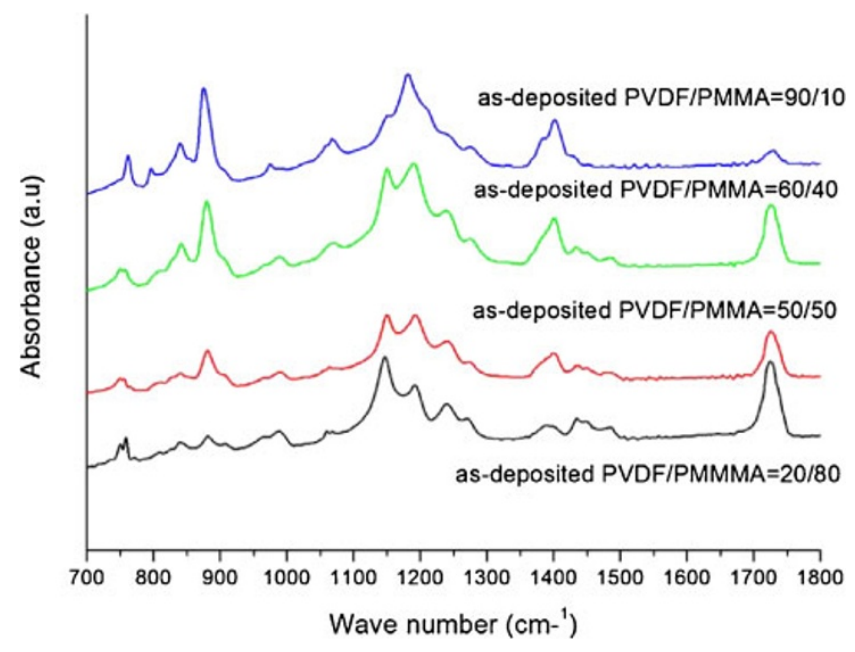

Figure 6. FT-IR spectra of as-deposited PVDF/PMMA nanofiber with various blending ratios.

and slow cooling from the melts. ${ }^{12}$ At the PVDF ratio higher than $50 \%$, on the other hand, the PVDF/PMMA blend nanofiber had a mixture of an amorphous and a crystalline state. In the second column of Table I, the reduced degree of crystallinity is shown, which was obtained from the total degree of crystallinity divided by the weight fraction of PVDF. The increases of the PVDF ratio in PVDF/PMMA blends increased both the total and reduced degree of crystallinities. In addition, at the PVDF blend ratio higher than $60 \mathrm{wt} \%$, the ESD process is effective for formation of crystalline structure of PVDF in the PVDF/PMMA nanofiber compared with the one for 50/50 or lower. The melting point of the PVDF/PMMA blend nanofiber substantially corresponded to the crystallinity.

Figure 6 shows FT-IR spectra of PVDF/PMMA blend nanofiber. The $\beta$-phase crystal of PVDF was observed in two vibration band peaks at around $840 \mathrm{~cm}^{-1}$ and $1278 \mathrm{~cm}^{-1}$. The $\alpha$-phase vibration band peak was observed at a peak around $766 \mathrm{~cm}^{-1}$. The $\beta$-phase crystal fraction of the PVDF/PMMA blend nanofiber estimated by the Salimi's approach ${ }^{16}$ for the blending ratio $20 / 80,50 / 50,60 / 40$ and $90 / 10$ were $0.43,0.47$, 0.47 , and 0.45 , respectively. Although the nominal ratio of the $\beta$-phase crystal of PVDF appears almost identical, the actual amount of $\beta$-phase crystal in the unit weight is increasing with the increase in the PVDF ratio (see Table I, the reduced amount of crystal region increases, as mentioned earlier). Figure 6 also shows the evidence of the hydrogen bonding between carbonyl group of PMMA and hydrogen atom of PVDF, where the vibration band peaks of the carbonyl groups were observed significantly at $1727 \mathrm{~cm}^{-1}$ or $1725 \mathrm{~cm}^{-1}$ for PVDF/PMMA $=20 / 80$ and 90/10 compared with the one for the pure PMMA at $1730 \mathrm{~cm}^{-1}$. These slight but significant shifts of carbonyl group were corresponds to the previous results of Saito et al. ${ }^{17}$

The crystalline structure of PVDF/PMMA blend nanofiber was analyzed by WAXD (Figure 7). At the low PVDF ratio, the amorphous state of PMMA shaded the crystal phase of PVDF. At the higher PVDF blend ratio, however, the WAXD

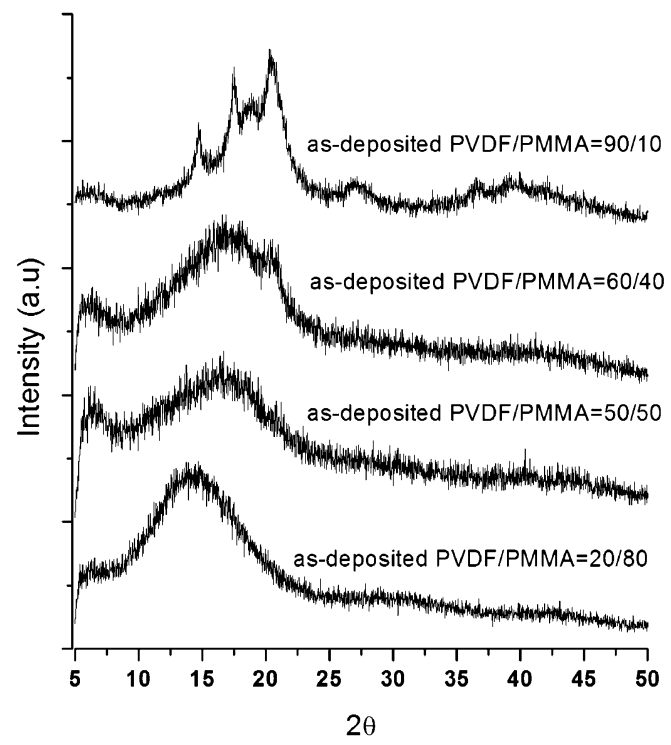

Figure 7. Wide angle X-ray diffraction diagram of as-deposited PVDF/ PMMA nanofiber with various blending ratios.

pattern of the PVDF crystal phase was observed very clearly, where PVDF/PMMA nanofibers have a mixture of $\alpha$ - and $\beta$-phase crystal structures. $\beta$-phase peak is around $20.3^{\circ}$ and $\alpha$-phase at peak around $17.7,18.4$ and 26 respectively. ${ }^{8,12-14}$ These results correspond to the one for the IR measurements, as mentioned earlier.

\section{CONCLUSIONS}

In the present study, PVDF/PMMA blend nanofibers were prepared by electrospray deposition (ESD) from the polymer solutions with various blending ratios and at various humidity conditions. The degree of crystallinity of the PVDF/PMMA blend nanofiber depended on the blending ratio. At low PVDF ratio, the nanofiber was amorphous. At high PVDF blend ratio, on other hand, the nanofiber showed a higher crystallinity (mixture of $\alpha$ - and $\beta$-phase crystal structures). In addition, ESD process enhanced crystal formation of PVDF for the blends of high PVDF ratio. High humidity reduced the formation of beaded fiber and increased the average fiber diameter. The PVDF/PMMA blend nanofiber has a great potential in the advanced filtration, and sensors and actuators.

Received: August 1, 2008 Accepted: January 28, 2009 Published: March 11, 2009

\section{REFERENCES}

1. Y. Dzenis, Science, 304, 1917 (2004).

2. M. M. Stevens and J. H. George, Science, 310, 5751, 1135 (2005).

3. J. Doshi and D. H. Reneker, J. Electrostat., 35, 151 (1995).

4. X. J. Zhi-Kang Xu, L. S. Wan, C. Innocent, and P. Seta, Macromol. Rapid Commun., 27, 1341 (2006).

5. T. Grafe and K. Graham, presented at INTC 2002: International Nonwovens Technical Conference (Joint INDA-TAPPI Conference), Atlanta, Georgia, September 24-26, 2002. 
6. V. A. Kirsh, Colloid J., 62, 714 (2000).

7. M. N. Lebedev, I. B. Stechkina, and A. L. Chernyakov, Colloid J., 62, 731 (2000).

8. M. Nasir, H. Matsumoto, T. Danno, M. Minagawa, T. Irisawa, M. Shioya, and A. Tanioka, J. Polym. Sci., Part B: Polym. Phys., 44, 779 (2006).

9. H. Kawai, Jpn. J. Appl. Phys., 8, 975 (1969).

10. Y. Ye, Y. Jiang, Z. Wu, and H. Zeng, Integrated Ferroelectrics, 80, 245 (2006).

11. W. Sae-lim, W. Tanthapanichakoon, and C. Kanaoka, J. Aerosol Sci.,
37, 228 (2006).

12. H. Horibe and M. Taniyama, J. Electrochem. Soc., 153, G119 (2006).

13. R. Gregorio, Jr. and N. C. P. S. Nociti, J. Phys. D: Appl. Phys., 28, 432 (1995).

14. J. Martínez-Salazar, J. C. C. Camara, and F. J. B. Calleja, J. Mater. Sci., 26, 2579 (1991).

15. S. D. Vrieze, T. V. Camp, A. Nelvig, B. Hagström, P. Westbroek, and K. D. Clerck, J. Mater. Sci., online published, (2008).

16. A. Salimi and A. A. Yousefi, Polym. Test., 22, 699 (2003).

17. H. Saito, Y. Fujita, and T. Inoue, Polym. J., 19, 405 (1987). 